\title{
Modeling Respiratory Motion for Cancer Radiation Therapy Based on Patient-Specific 4DCT Data
}

\author{
Jaesung Eom ${ }^{1}$, Chengyu $\mathrm{Shi}^{2}$, Xie George $\mathrm{Xu}^{1}$, and Suvranu $\mathrm{De}^{1}$ \\ ${ }^{1}$ Department of Mechanical, Aerospace and Nuclear Engineering, \\ Rensselaer Polytechnic Institute, Troy, NY 12180, USA \\ \{eomj, xug2, des\} @rpi.edu \\ ${ }^{2}$ Department of Radiation Oncology, \\ University of Texas Health Science Center at San Antonio, San Antonio, TX 78229, USA \\ shic@uthscsa.edu
}

\begin{abstract}
Prediction of respiratory motion has the potential to substantially improve cancer radiation therapy. A nonlinear finite element (FE) model of respiratory motion during full breathing cycle has been developed based on patient specific pressure-volume relationship and 4D Computed Tomography (CT) data. For geometric modeling of lungs and ribcage we have constructed intermediate CAD surface which avoids multiple geometric smoothing procedures. For physiologically relevant respiratory motion modeling we have used pressure-volume (PV) relationship to apply pressure loading on the surface of the model. A hyperelastic soft tissue model, developed from experimental observations, has been used. Additionally, pleural sliding has been considered which results in accurate deformations in the superior-inferior (SI) direction. The finite element model has been validated using 51 landmarks from the CT data. The average differences in position is seen to be $0.07 \mathrm{~cm}(\mathrm{SD}=0.20 \mathrm{~cm}), 0.07$ $\mathrm{cm}(0.15 \mathrm{~cm})$, and $0.22 \mathrm{~cm}(0.18 \mathrm{~cm})$ in the left-right, anterior-posterior, and superior-inferior directions, respectively.
\end{abstract}

\section{Introduction}

Respiratory motions have a profound impact on the radiation treatment planning of cancer in the lung and adjacent tissues. In external beam radiation treatment, for example, a lethal radiation dose is delivered through precisely conformed radiation to the target. The current radiation treatment paradigm, however, is largely based on an assumption that both tumor location and shape are well known and remain unchanged during the course of radiation delivery. Such a favorable rigid-body relationship does not exist in anatomical sites such as the thoracic cavity and the abdomen, owing predominantly to respiratory motions. When the tumor-bearing normal organs move during radiation therapy, discrepancies between planned and actually delivered radiation doses can be quite significant. As a result, although higher radiation doses have shown better local tumor control, organ motions have sometimes required less aggressive treatment strategies having relatively large dose margins to tolerate potential targeting errors. 
One previous approach to account for respiration caused target movement is to consider a larger planning target volume which covers a composite of 3D volumes of the moving target defined by the entire respiratory cycle. A relatively new approach is based on an image-guided technique which aligns and delivers the radiation according to a gated time and position or follows the tumor's trajectory during the respiratory cycle, to allow for a smaller and more conformal treatment volume. Hence, it is important to be able to predict the pattern of the lung motion as part of radiation therapy and know the tumor location in real time.

Discrepancies between the Deformable Image Registration (DIR) and physics based modeling methods are apparent when comparing motion field estimates. The question arises as to what motion field is more realistic and physiologically correct. There are several attempts to include the physiology in non-linear registration based methods [1, 2]. The basic assumptions of DIR often concern image-related aspects, and hence physiological and anatomical processes are not taken into consideration. As a result, gray values of anatomically corresponding voxels are treated to be constant over time. More accurate physics based techniques have also been reported more recently [3-5]. However, in most of the existing models the physiological data is ignored both in applying the boundary conditions and in using appropriate material models for the lung tissue.

To investigate these issues, a patient-specific non-linear finite element (FE) lung model is developed in this study by considering vigorous physiological conditions in the modeling. During inspiration, the diaphragm and the external intercostals contract with each other, resulting in an increased the thoracic volume. The resulting decrease in alveolar pressure causes the air to enter the lung. Expiration, on the other hand, is passive and the diaphragm relaxes, leading to a reduced thoracic volume and an increased pressure. The chest pressure-volume (PV) curve can be constructed by plotting lung volumes against pleural pressures that are estimated from esophageal pressures and body plethysmography [6]. Such PV curve data is used to drive the lung motion which simulates the breathing. Additionally, we take advantage of the sophisticated Computer Aided Engineering (CAE) concept in the geometric modeling of organs. The CAD surface reconstruction procedure affords more interactive mesh control and preserves the original geometric features of organs obtained from 4D CT image data.

This paper introduces the CAD surface reconstruction procedure using 4D CT data and then briefly discusses the outline of a nonlinear finite element modeling including the application of boundary and contact/sliding conditions. The advantage of the proposed geometric modeling procedure over conventional smoothing for the purposes of improving the accuracy of the respiratory simulation is presented.

\section{Methods and Materials}

\subsection{Geometric Modeling}

4D respiration gated CT images were acquired using a 16 Slice Brilliance CT Big Bore Oncology configuration (Philips). Breathing information was obtained using the associated Pneumo Chest bellows (Lafayette Instruments). Each Image slice has a resolution of $0.98 \mathrm{~mm} \times 0.98 \mathrm{~mm}$ and a thickness of $2 \mathrm{~mm}$. The categorized 10 phases of images were contoured into different ROIs (regions of interest) as [7]. According to the volume of each phase, the end of expiration (EE) state and the end of inspiration 




Fig. 1. Geometric modeling procedure using the CAD surface reconstruction (Reverse engineering)

(EI) states were selected. For accurate simulations, a uniform mesh of "good quality" must be used. However, for patient-specific geometric modeling, the characteristic features of the 4D CT image data must be preserved. It is noted that, in most previous studies $[4,5,8]$, combination of mesh decimation and Laplacian smoothing were used to reduce the number of elements from the highly dense but non-regular mesh to the more uniform mesh acceptable in FE simulations. The problem of this procedure is that details of the mesh topology are lost. Also, the procedure is not interactive as multiple steps are involved. In this study, we used a CAD surface reconstruction approach to convert the ROIs into FE meshes as depicted in Figure 1. Primary surfaces are generated from ROI contour lines in Rhinoceros 3D (Robert McNeel \& Associates, Seattle, WA). From these surfaces, NURBS-based CAD surfaces are reconstructed and converted into suitable FE meshes using HYPERMESH (Altair Engineering, Troy, MI). The surface that closely fits the tessellated surface is generated. This approach greatly simplifies the procedure of FE mesh generation and from CT scanned image data without losing the geometric details.

\subsection{Physiologically-Based Respiratory Motion Modeling}

The motion of the lungs during inhalation is physiologically caused by the expansion of the thoracic cavity. This expansion is induced by contraction of the diaphragm and outer intercostal muscles. These movements cause the pressure to change in the pleural cavity surrounding the lungs and the alveoli and, as a result, the air flows from the atmosphere into the lungs. This in turn causes a change in the intrapleural pressure which exerts force on the lung surface. Hence, the lung expands and the visceral pleura slides against the internal surface of the thoracic cavity with nearly frictionless contact due to lubrication of pleural liquid [9].

To model the motion of the lungs between EE to EI, a quasi-static nonlinear finite element model has been developed. A distributed time-varying pressure load was applied to the surface of the geometric lung model. The current paper outlines a physiologically-based modeling approach. The pressure amplitudes have been computed by comparing CT image data and FEM results. For now, the pressure history was assumed to follow the sinusoidal curve [10] according to the body plethysmography and the 
parameterized P-V curve [11]. We limited the expansion to a geometry defined by the lung shape at the end of inspiration phase. Pleural sliding is treated as a contact-friction model. Any contact between the lungs and the ribcage is modeled without friction-an approach that is justified as there is an incompressible and friction-minimizing pleural fluid in and between the visceral and parietal pleural.
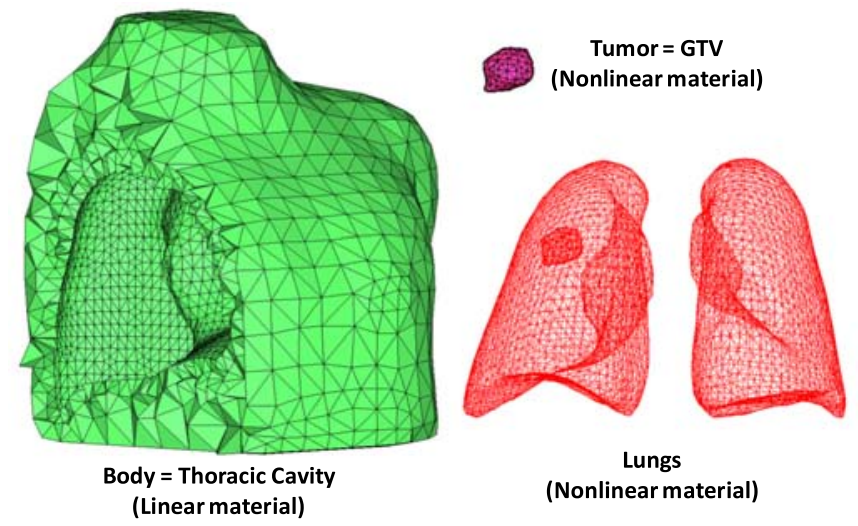

Fig. 2. Finite element models including a thoracic cavity, a tumor (gross target volume for radiation treatment purposes) and lungs

The FE model, which has 66704 tetrahedral elements and 55947 degrees of freedom in total, is composed of the thoracic cavity and the lungs with an embedded tumor in the right lung as seen in Figure 2. The lungs and thoracic cavity are fixed at the root of lungs according to anatomy.

The lung tissue was modeled as a hyperelastic material with the following expression for the strain energy per unit volume [12]

$$
\begin{aligned}
\rho_{0} W & =\frac{1}{2} c \exp \left(a_{1} E_{x x}^{2}+a_{2} E_{y y}^{2}+2 a_{4} E_{x x} E_{y y}\right)+\frac{1}{2} c \exp \left(a_{1} E_{x x}^{2}+a_{2} E_{z z}^{2}\right. \\
& \left.+2 a_{4} E_{x x} E_{z z}\right)+\frac{1}{2} c \exp \left(a_{1} E_{z z}^{2}+a_{2} E_{y y}^{2}+2 a_{4} E_{z z} E_{y y}\right)
\end{aligned}
$$

where $c, a_{1}, a_{2}, a_{4}$ are material constants derived from experiments, and $E_{\mathrm{xx}}, E_{\mathrm{xy}}$ etc. are the components of the Green strain. For simplicity, lung tissue was assumed to be homogeneous and thoracic cavity was assumed to be linear elastic material $(\mathrm{E}=$ $6.0 \mathrm{kPa}$ Poisson's ration $=0.4$ from [5]). Simulations, using Abaqus (Dassault Systèmes Simulia Corp., Providence, RI), have been carried out on an Intel Core2 Quadcore 2.83 GHz CPU machine with $8 \mathrm{~GB}$ RAM for 2.1 hours.

\section{Results and Discussion}

\subsection{Comparison on Laplacian Smoothing vs. CAD Surface Reconstruction}

To assess the advantage of the CAD surface reconstruction procedure over conventional mesh preparation using multiple Laplacian smoothing and decimating [6], we 
compared the geometric quality of the elements. Four mesh quality indices, commonly used in CAE [13] are used:

(a) Aspect ratio: This is the ratio of the longest edge of an element to its shortest edge.

(b) Maximum and minimum interior angles: These maximum and minimum values are evaluated independently for triangle facet.

(c) Jacobian: This measures the deviation of an element from its ideal or "perfect" shape, such as a triangle's deviation from equilateral. The Jacobian value ranges from 0.0 to 1.0 , where 1.0 represents a perfectly shaped element. The determinant of the Jacobian relates the local stretching of the parametric space which is required to map it to the global coordinate space.

In Figure 3, elements which violate the mesh quality indices for each approach are color-coded. The threshold for violation is 0.9 for Jacobian, 2.0 for aspect ratio, $80^{\circ}$ and $50^{\circ}$ for the maximum and minimum interior angle, respectively. The volume change under modeling procedure can indicate the loss of geometric feature. It is clear
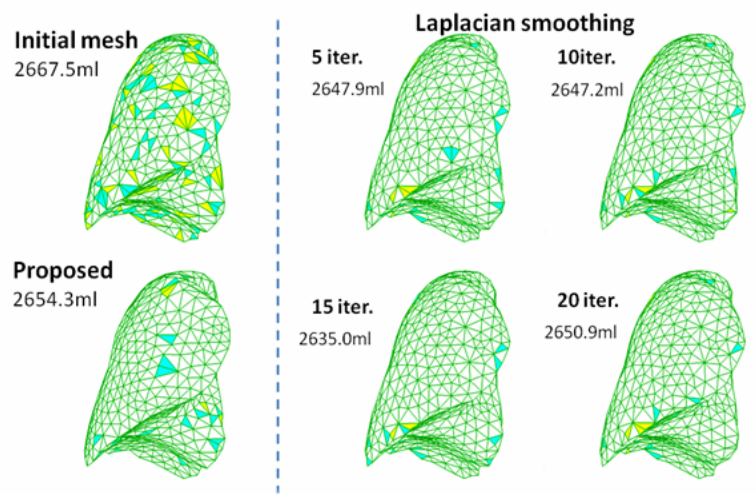

Fig. 3. Comparison of relaxing (Laplacian smoothing) vs. proposed CAD surface reconstruction procedure showing changes in mesh volume and elements that are close to or violating the quality indices using the following color coding : light blue = warning that an index is close to threshold and yellow $=$ failure.

Table 1. Percentage of elements violating threshold criteria

\begin{tabular}{lccll}
\hline Mesh & Jacobian (0.9) & Aspect Ratio (2.0) & $\begin{array}{l}\text { Max angle } \\
\left(>80^{\circ}\right)\end{array}$ & $\begin{array}{l}\text { Min angle } \\
\left(<50^{\circ}\right)\end{array}$ \\
\hline Initial & $21.1 \%$ & $0.2 \%$ & $49.9 \%$ & $83.7 \%$ \\
\hline Laplacian Smoothing & & & \\
& & & & \\
5 Iterations & $4.2 \%$ & $0.2 \%$ & $28.3 \%$ & $66.9 \%$ \\
10 Iterations & $4.1 \%$ & $0.2 \%$ & $27.7 \%$ & $66.0 \%$ \\
15 Iterations & $3.4 \%$ & $0.2 \%$ & $24.7 \%$ & $63.7 \%$ \\
20 Iterations & $3.9 \%$ & $0.2 \%$ & $27.6 \%$ & $66.2 \%$ \\
\hline Proposed model & $3.0 \%$ & $0.0 \%$ & $21.3 \%$ & $59.3 \%$ \\
\hline
\end{tabular}


that the proposed geometric modeling procedure preserves initial geometry of the CT scan data. Laplacian smoothing is an algorithm to smooth a polygonal mesh. For each vertex in the mesh, a new position is chosen based on local information (such as the position of neighbors) and then the vertex is moved. Laplacian smoothing focuses on moving point locations to improve triangulation without any guarantees on the preservation of the original geometric features.

In previous studies, 10 iterations of Laplacian smoothing and additional 10 smoothing iterations with decimation were used to prepare the computational mesh[5, 8]. Table 1 shows that 10 cycles of smoothing iterations reduce the original volume by $20.3 \mathrm{ml}$ and the relaxing operations fail to enhance the mesh quality indices, while our proposed approach has achieved better meshes with $13.2 \mathrm{ml}$ of volume loss.

\subsection{Validation Based on Landmarks}

The procedure to evaluate the modeling accuracy was based on the patient specific models and 4D CT image data. As shown in Figure 4, anatomical points that represent the bifurcation of vessels and airways were chosen on the exhale and inhale images. A total of 51 such landmarks were chosen from the CT images. The motion of the corresponding points in the FE model provides a measure of our modeling accuracy.
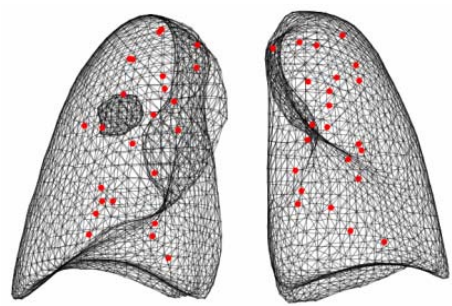

Fig. 4. Landmark bifurcation positions inside the lungs determined by radiologists

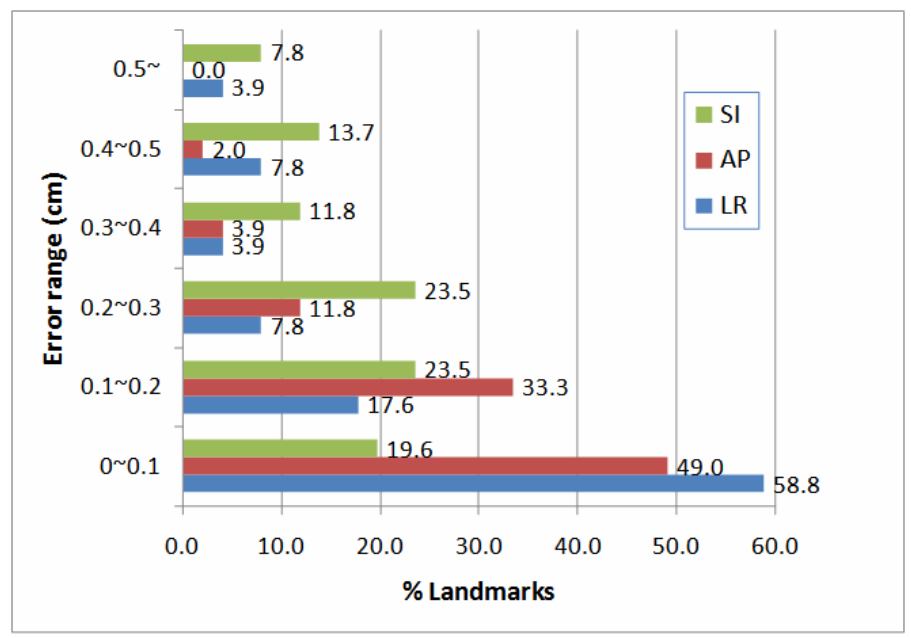

Fig. 5. Histogram of errors at landmarks at end of inhalation 
We observe that the mean deviations $\left(P_{F E M}-P_{C T}\right)$ of our FE model predictions from the CT data are $0.07 \mathrm{~cm}(\mathrm{SD}=0.20 \mathrm{~cm}), 0.07 \mathrm{~cm}(\mathrm{SD}=0.15 \mathrm{~cm})$, and $0.22 \mathrm{~cm}$ $(\mathrm{SD}=0.18 \mathrm{~cm})$ in left-right $(\mathrm{LR})$, anterior-posterior $(\mathrm{AP})$, and superior-inferior (SI) directions, respectively. On the majority of landmarks the displacement errors are less than $2 \mathrm{~mm}$ (Figure 5). It should be noted that the landmarks are located in regions where effects of heterogeneity of the lung tissue are not negligible. Considering this, the accuracy of our homogeneous model is remarkable.

\section{Conclusions}

We present a nonlinear finite element model of respiratory motion during full breathing cycle based on patient-specific pressure-volume relationship and 4D CT data. For geometric modeling of the lungs and ribcage we have constructed an intermediate CAD surface between 4D CT scanned images and meshes for FE computation. This avoids multiple geometric smoothing procedures and increases the quality of the FE mesh while preserving geometric features of the CT scans. For a patient-specific FE lung model we have used pressure-volume (PV) relationship of lungs as physiological conditions and hyperelastic soft tissue model [12]. The PV relationship provides physiologically relevant boundary conditions over the entire breathing cycle. Validation using 51 landmarks from the CT image has been performed and our proposed model shows excellent agreement with CT data with a position error of less than 2 $\mathrm{mm}$ for most of the landmarks.

Acknowledgments. The authors would like to gratefully acknowledge the funding support from NIH/NLM grant R01LM009362.

\section{References}

1. McClelland, J., Blackall, J., Tarte, S., Chandler, A., Hughes, S., Ahmad, S., Landau, D., Hawkes, D.: A continuous 4D motion model from multiple respiratory cycles for use in lung radiotherapy. Medical Physics 33, 3348 (2006)

2. Sarrut, D., Boldea, V., Miguet, S., Ginestet, C.: Simulation of four-dimensional CT images from deformable registration between inhale and exhale breath-hold CT scans. Medical Physics 33, 605 (2006)

3. Zhang, T., Orton, N.P., Mackie, T.R., Paliwal, B.R.: Technical note: A novel boundary condition using contact elements for finite element deformable image registration. Medical Physics 31, 2412-2415 (2004)

4. Al-Mayah, A., Moseley, J., Brock, K.: Contact surface and material nonlinearity modeling of human lungs. Physics in Medicine and Biology 53, 305 (2008)

5. Brock, K., Sharpe, M., Dawson, L., Kim, S., Jaffray, D.: Accuracy of finite element model-based multi-organ deformable image registration. Medical Physics 32, 1647 (2005)

6. West, J.: Respiratory Physiology: The Essentials. Williams \& Wilkins (2007)

7. Lin, L., Shi, C.T., Liu, Y., Swanson, G., Papanikolaou, N.: Development of a novel postprocessing treatment planning platform for 4D radiotherapy. Technology in Cancer Research \& Treatment 7, 125-132 (2008) 
8. Villard, P., Beuve, M., Shariat, B., Baudet, V., Jaillet, F.: Lung mesh generation to simulate breathing motion with a finite element method. In: Proceedings of Eighth International Conference on Information Visualisation. IV 2004, pp. 194-199 (2004)

9. D’Angelo, E., Loring, S., Gioia, M., Pecchiari, M., Moscheni, C.: Friction and lubrication of pleural tissues. Respiratory Physiology \& Neurobiology 142, 55-68 (2004)

10. Lujan, A.E., Larsen, E.W., Balter, J.M., Ten Haken, R.K.: A method for incorporating organ motion due to breathing into 3D dose calculations. Medical Physics 26, 715-720 (1999)

11. Santhanam, A.: Modeling, Simulation, And Visualization of 3d Lung Dynamics. University of Central Florida Orlando, Florida (2006)

12. Zeng, Y., Yager, D., Fung, Y.: Measurement of the mechanical properties of the human lung tissue. Journal of Biomechanical Engineering 109, 169-174 (1987)

13. Bathe, K.: Finite element procedures. Englewood Cliffs, New Jersey (1996) 